\section{Crystal Scanner for Nano-Metrology Applications}

Paul West, Zhiqiang Peng, Natalia Starostina Pacific Nanotechnology, Inc. pwest@pacificnanotech.com

\section{Introduction}

Traditionally a scanning probe microscope (SPM), such as the atomic force microscope (AFM), affords spectacular images of surfaces at the nanometer scale. With advanced developments in scanner design, probe manufacturing and force sensor technology it is now possible to make quantitative metrological measurements with an SPM. Quantitative metrological measurements that are

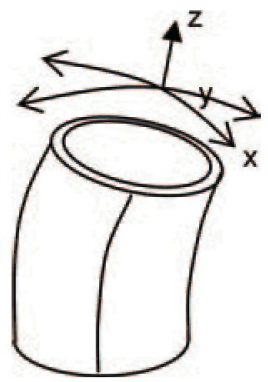

Figure 1: A piezoelectric ceramic in the shape of a tube is often used in AFMs for generating 3-D motion. Electrodes are deposited on the inner and outer surface of the tube. By sectioning the outer electrode in four quadrants, and using the inner electrode, 3-D motion is possible. There is a variant of the tube scanner design that has a separate $Z$ ceramic at the end of the tube; however, there are serious coupling issues between all three of the axis in all tube scanner designs.

possible include: a) dimensional measurements of micro/nano fabricated structures, b) surface texture of surfaces having RMS values of only a few angstroms, and c) measurements of the number of grains, and particles on a surface as well as grain and particle sizes, areas, volumes, and distributions.

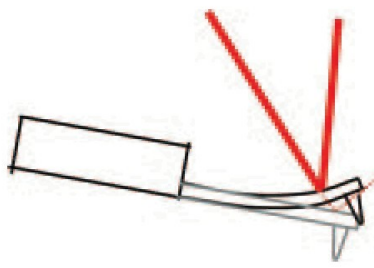

Figure 2: In the light lever force sensor a laser beam is reflected of the backside of a cantilever. There are two problems with this approach, first the cantilever is at an angle to the surface, and the deflection of the cantilever causes a change in the probe' sample angle.

The design of the crystal scanner is optimized for metrological measurements. The force sensor is small and permits use of advanced flexure stages, which are orthogonal and have minimal out of plane motion. Further, the probes used in the crystal scanner may be constructed from any type of material such as tungsten, silicon, or even glass.

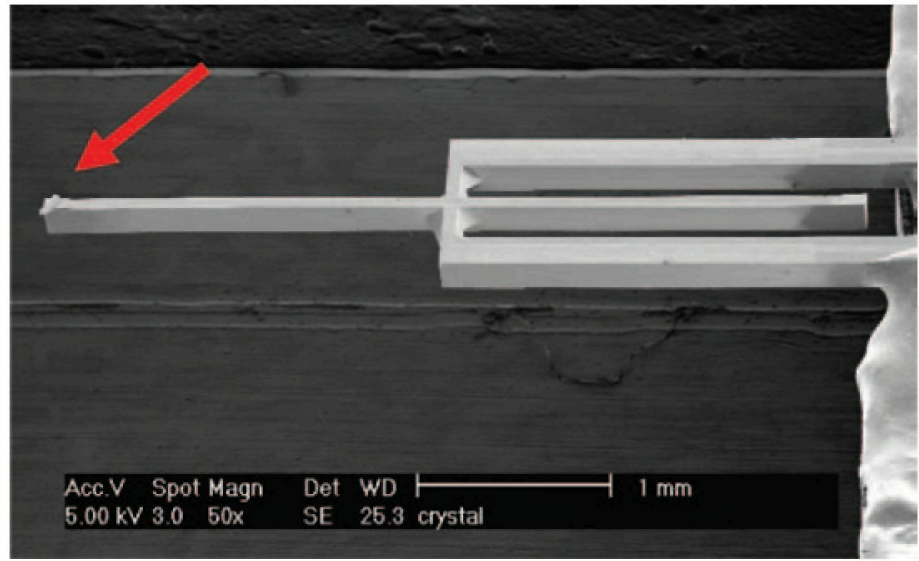

Figure 3a: This SEM image shows the cross quartz crystal resonator used in the crystal sensor. A cantilever, identified by the red arrow, is mounted at the end of one of the resonating beams of the quartz oscillator. The dimensions of the oscillating beams are: $2.1 \mathrm{~mm}$ long, $0.09 \mathrm{~mm}$ wide, and $0.17 \mathrm{~mm}$ thick. Electrodes are deposited on two opposing faces of the beams.
Background

Scanners that move the probe in an AFM are typically constructed with piezoelectric ceramics. The three dimensional motions of the scanner are made possible by configuring the ceramics in one of several geometries such as a tube or a
Figure 3b: The crystal sensor is held in the scanner with a zero insertion force mechanism. The crystal change tool is used to remove the force of springs that hold the crystal sensor in the scanner. Changing the crystal sensor takes less than a minute.
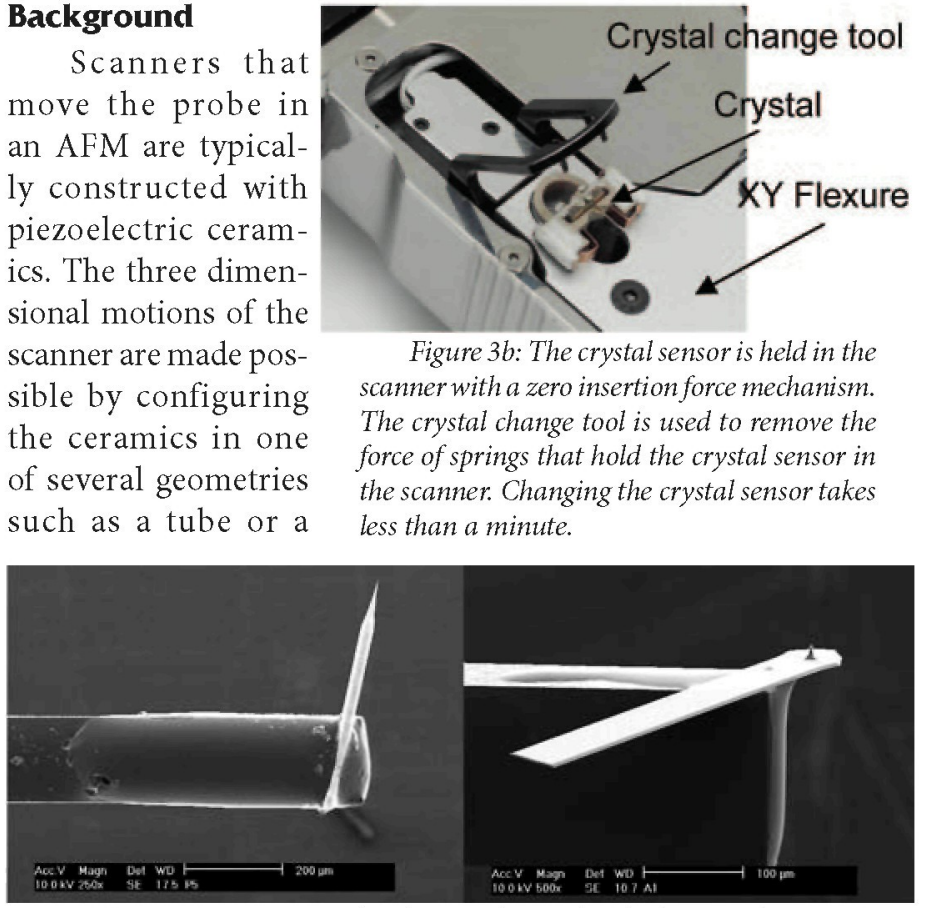

Figure 4: Many types of probes may be used with the crystal sensor. At the right is a tungsten probe and at the left is a silicon probe.

tripod. Regardless of the scanner configuration there are problems resulting from the hysterisis and creep of the piezoelectric ceramics. These effects make it difficult to make precise and accurate measurements in all areas of a scanner's field of view. Also, it is tedious to zoom in on a small feature in an initially-viewed, AFM large scan area image.

Typically, scanners suffer from crosstalk between the spatial axes scanned. For example, there is unwanted crosstalk between the $X Z, Y Z$, and $X Y$ axis, see figure 1. Crosstalk is possible even when the scan axes are fully linear and calibrated. This crosstalk makes the measurement of sidewall angles in structures such as semiconductor devices and DVDs impractical.

Finally, because scanners are designed from tripods or tubes, there is a considerable background bow in the images. Such bow may be removed from images with image processing software;

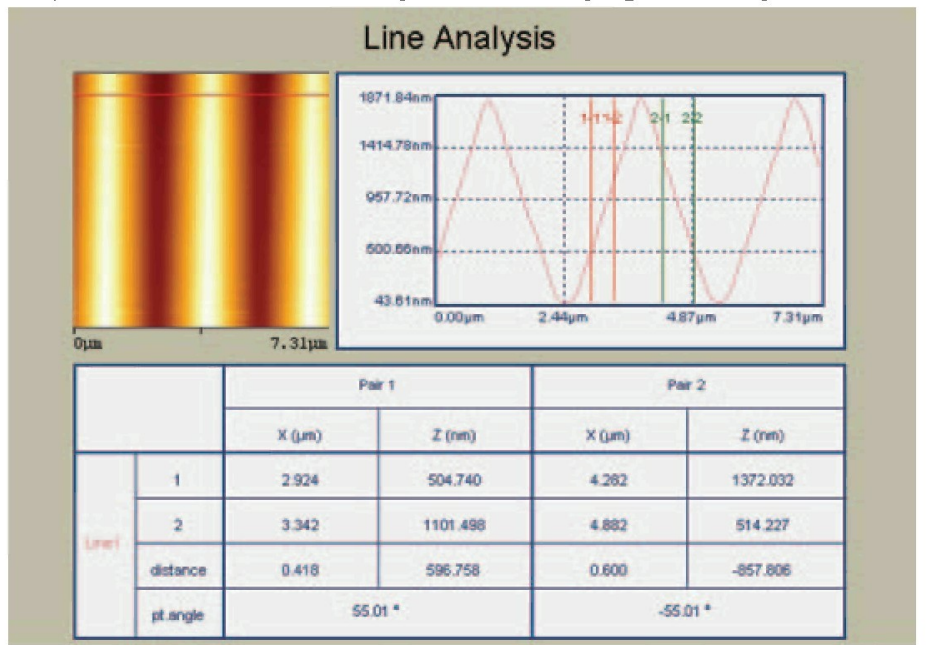

Figure 5a: $X Z$ and $Y Z$ crosstalk is tested using a reference sample having a triangle pattern etched its surface. The angles of the sides of the triangle patterns should be the same, 55 degrees. The line analysis at the right shows the correct angle is measured for both sides of the triangle with the Crystal Scanner. 


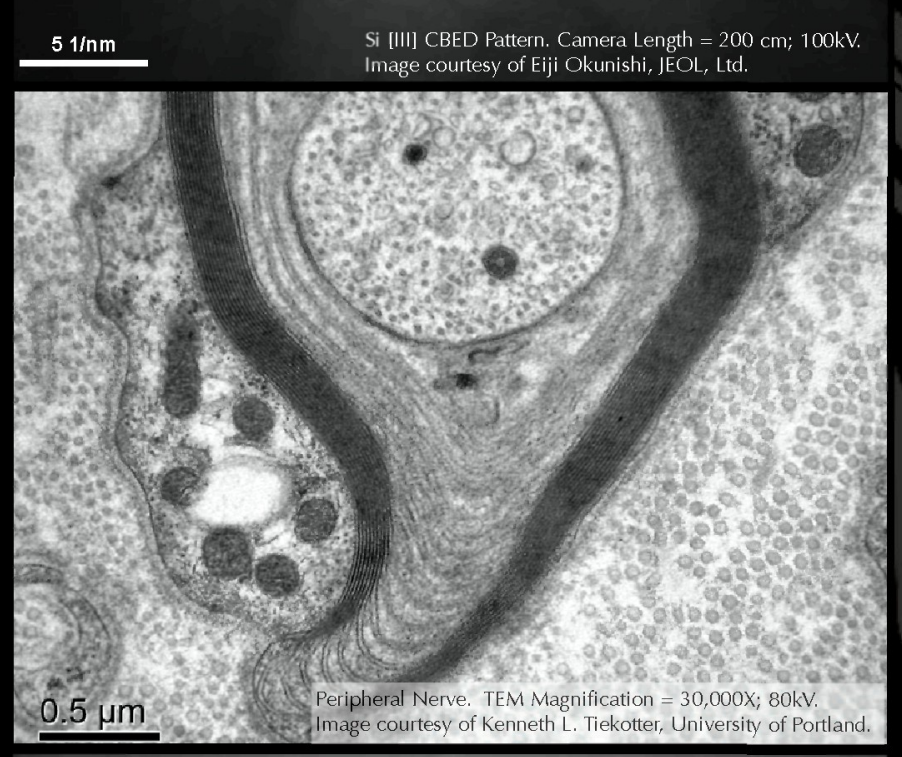

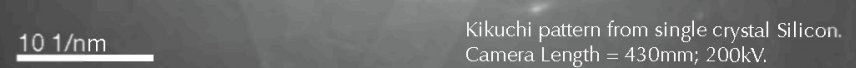

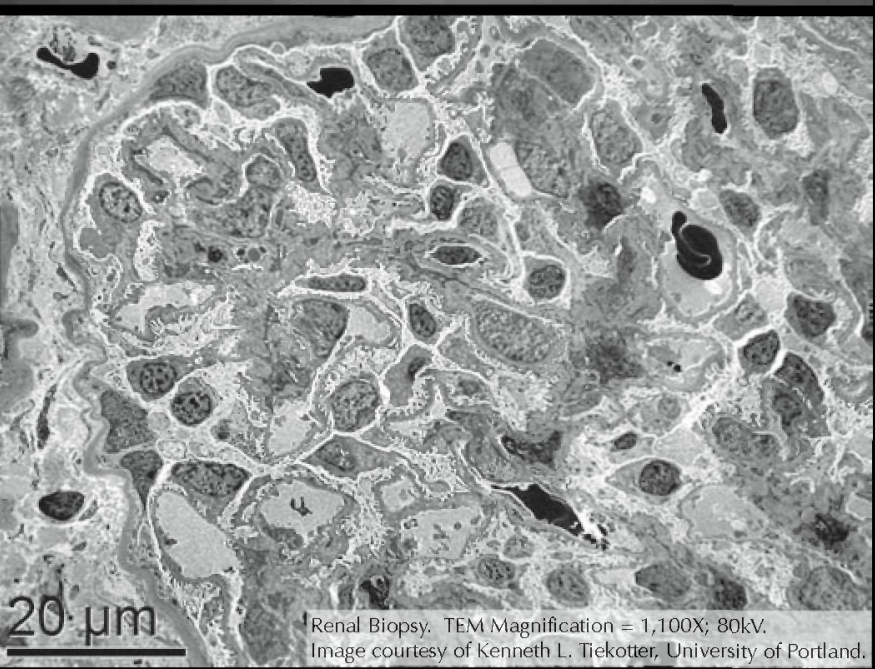

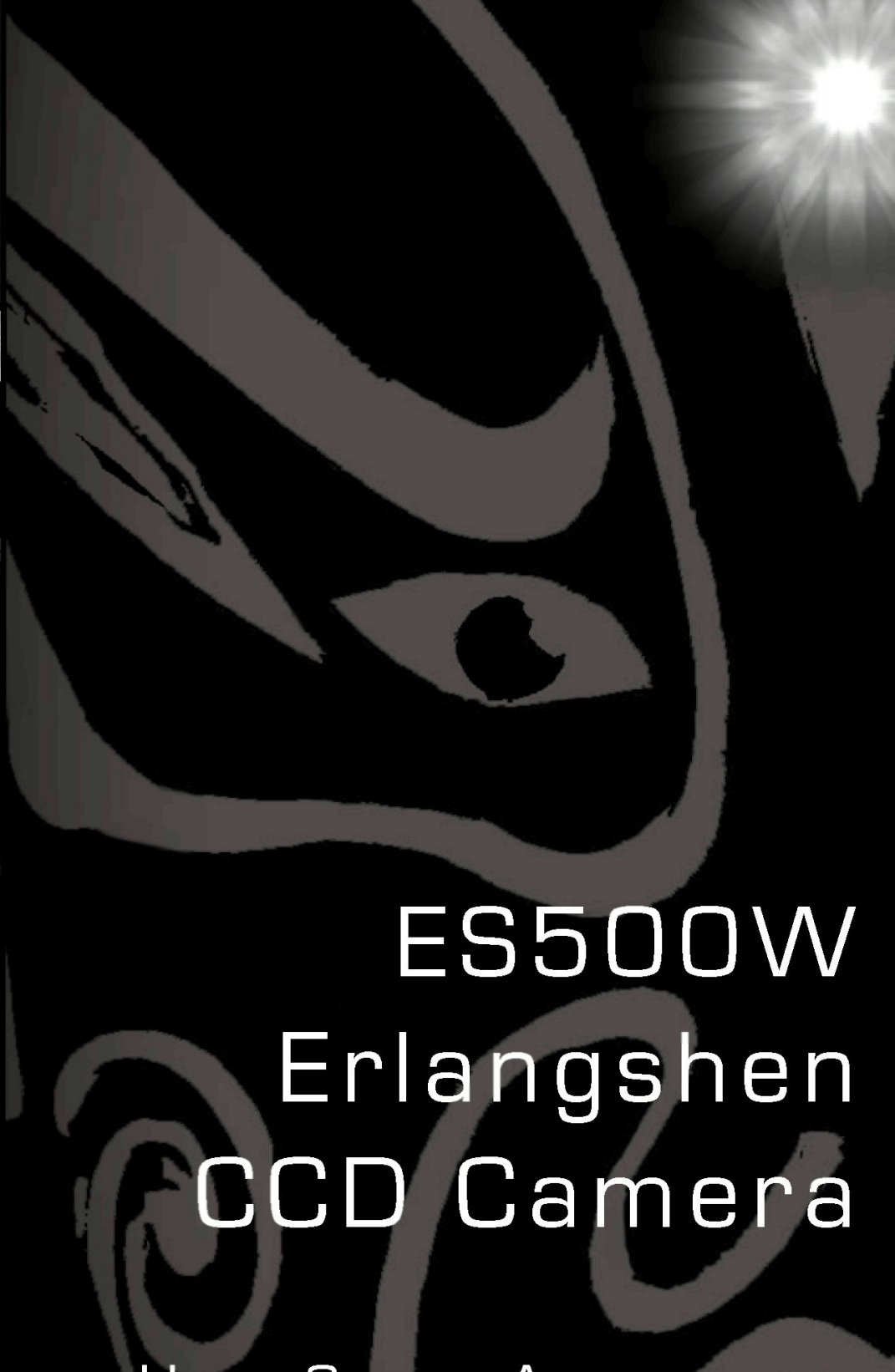

High Speed Affordable - Digital IMAging for TEM 

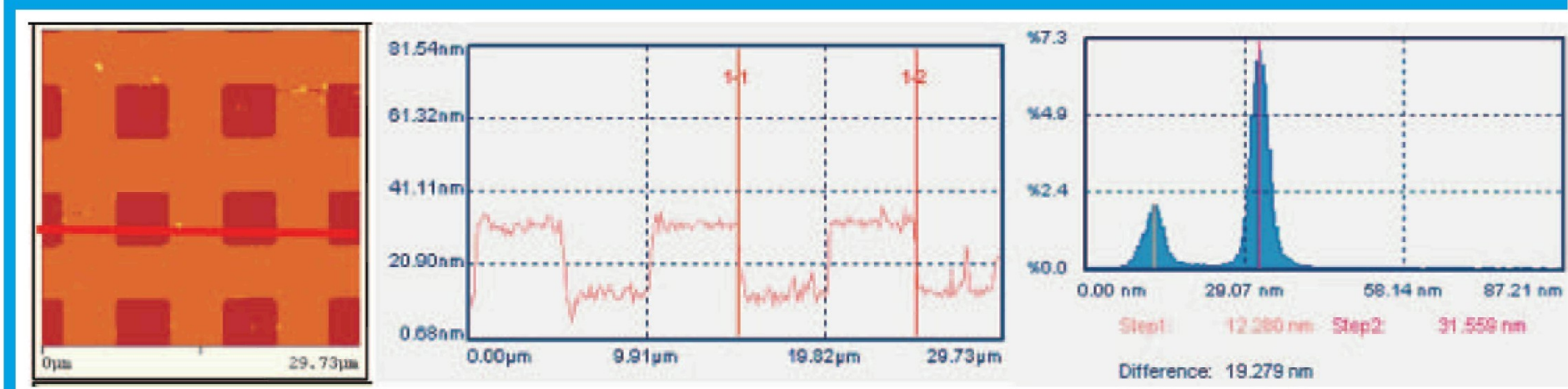

Figure 5b: The calibration in the X-Y axis is verified with a VLSI standard test pattern. The pitch of the standard is measured with a line profile and the restilt must be within the error margin of the standard. The $Z$ axis is calibrated by measuring the height of features in the standard. A histogram analysis is optimal because the histogram includes the data points from the entire image. however, such removal can easily add unwanted image processing artifacts.

In many of the first designs of scanning probe microscopes, the sample was scanned while the probe was held fixed. This approach was abandoned because scanning the sample greatly limited the number of sample types that could be scanned. Samples of different weight and sizes required different scan conditions. These unwanted problems are avoided when the probe is scanned in the $x, y$ and $z$ axis and the sample is held stationary.

X-nonlinearity, VLSI

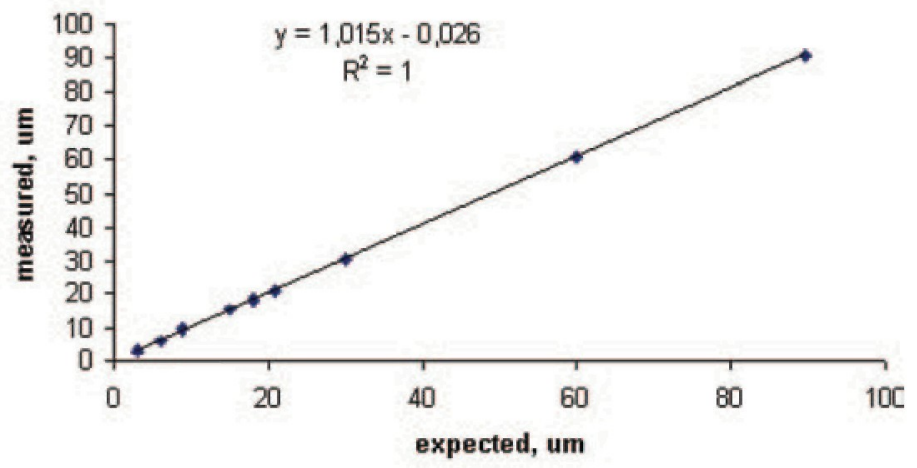

Figure $5 c X$ and $Y$ nonlinearity are determined by comparing the expected position of features on a test pattern with the measured position of features on the test pattern. This figure shows the results form a measurement of the features in the X axis of a VLSI standard.

Probes mounted at the end of a cantilever play a critical role in the ability to make metrology measurements with an atomic force microscope, see figure 2 . With the most common type of force sensor, the light lever, the angles between the cantilever and sample is established by the optical path of the light lever sub-system. Typically the probes are at fixed angle, between 5 and 15 degrees, with respect to the surface. Specialized designs have a probe that is mounted at an angle to compensate for cantilever/surface angle. Both types of cantilever/probe result in errors in metrological measurements with an AFM.

\section{Crystal Scanner ${ }^{\mathrm{TM}}$}

The crystal scanner is designed for making accurate and precise topographic images at the nano-scale. The crystal scanner has two major sub-components: the crystal sensor and the xyz piezoelectric scanner.

Crystal Sensor- The crystal sensor, shown in figure 3, is constructed using a quartz crystal oscillator having a resonance frequency at approximately $640 \mathrm{kHz}$. Forces between the probe and surface are detected by measuring a change in the vibration

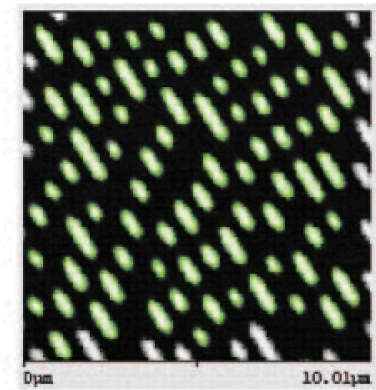

\begin{tabular}{|c|c|c|c|c|c|}
\hline-1 & Length (yn) & Wath (um) & Depth (m) & TopAngle & A \\
\hline 1 & 0.057 & 0.460 & 175.564 & -46.375 & \\
\hline 2 & 0.655 & 0.465 & 175.354 & 49.611 & \\
\hline 3 & 0.455 & 0.385 & 174 mat & 43.160 & \\
\hline 4 & 0.679 & 0.468 & 173213 & 48.919 & \\
\hline 5 & 0.720 & 0.482 & 171.928 & -44.143 & \\
\hline 6 & 0.456 & 0.356 & 174.912 & 46.973 & \\
\hline 7 & 0.444 & 0.349 & 181.122 & -40.220 & \\
\hline$\theta$ & 0.627 & 0.466 & 172.058 & 48.091 & \\
\hline 9 & 0.452 & 0.560 & $1 \mathrm{BT} .906$ & 45.601 & \\
\hline 10 & 0.526 & 0.459 & 170.583 & 47.205 & \\
\hline 11 & 0.461 & 0.370 & 170918 & 46.169 & \\
\hline 12 & 0.575 & 0.450 & 172.981 & 48.820 & \\
\hline 13 & 0.760 & 0.490 & 168.420 & -38.304 & \\
\hline 14 & 0.458 & 0.389 & 173594 & -47.519 & \\
\hline & n that & ก วคด & 170720 & $47 \pi$ & \\
\hline & & & & & \\
\hline
\end{tabular}

Figure 6a: The Crystal Scanner can measure high resolution images of DVD masters, stampers, and replicas. With specialized software the dimensions of each of the DVD bits are easily calculated.
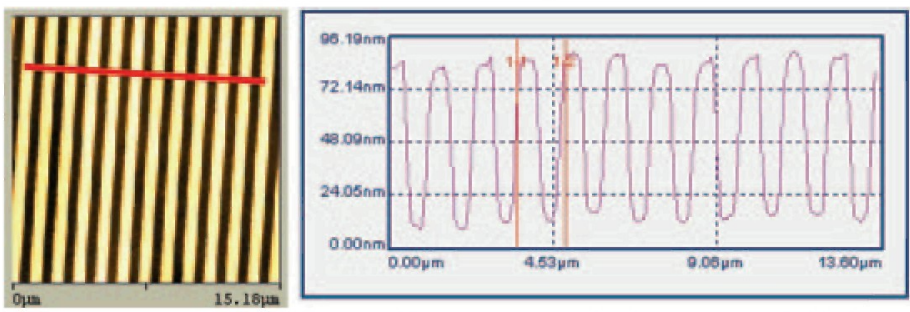

Figure $6 b$ - Single line profiles or automated software is available for measuring pitch of optical media using the Crystal Scanner.

amplitude or phase of the resonating crystal. In typical operation, the vibration amplitude of the crystal is $<0.4 \mathrm{~nm}$ and the probe/ surface forces are $<300 \mathrm{pN}$.

The damping force between the probe and surface are a complex combination of vertical and horizontal forces. In operation, the probe in the crystal sensor vibrates horizontally in the average plane of the surface. However, because of surface topography, the probe vibrations are damped by the vertical component of the interaction vector. Additionally, the probe vibration damping can occur from interactions with the contamination layer at the surface of a sample.

With the crystal sensor, topography images for all types of samples are measurable and provide exceptional visualization.

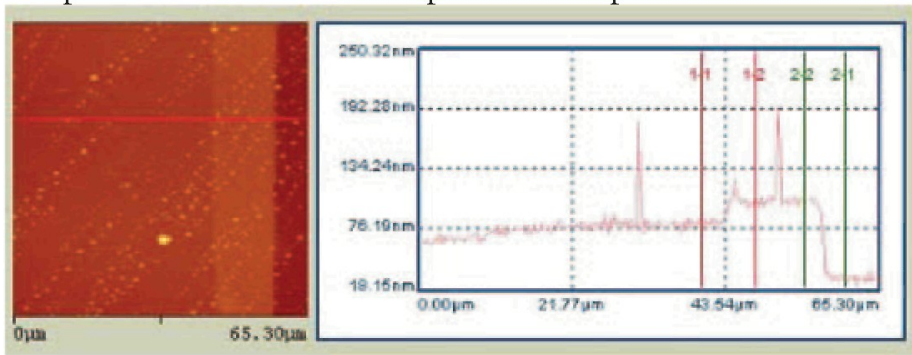

Figure 7 The step height of this structure on a flat panel display is measured with the Crystal Scanner. The step at the left is $20 \mathrm{~nm}$ and at the right is $80 \mathrm{~nm}$. 

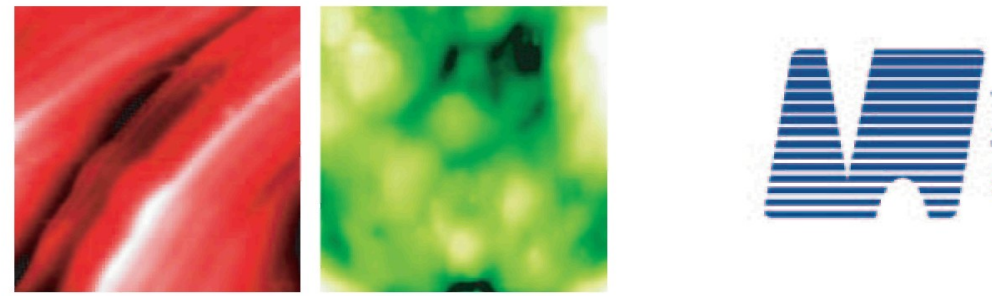
$\frac{\text { PACIFIC NANOTECHNOLOGY }}{\text { advancing nanotechnology }}$

\section{Nano-R ${ }^{\mathrm{TM}}$ Crystal Scanner ${ }^{\mathrm{TM}}$}
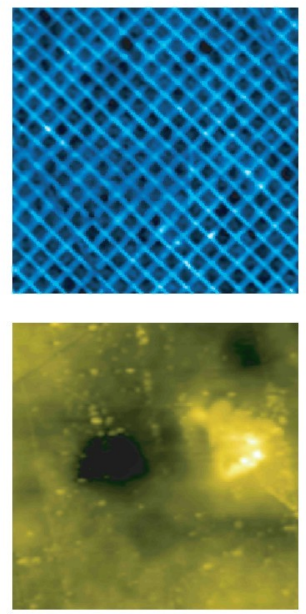

$1 \because \cdots, 1,1,1$ $\because, 11,1,1 \% 1$ ? $1,1,111$

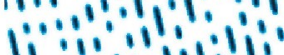
$11,211,19,1,11$ $1,1,1,1,1,9$ $1,1,1,1, \ldots 1,1,1$
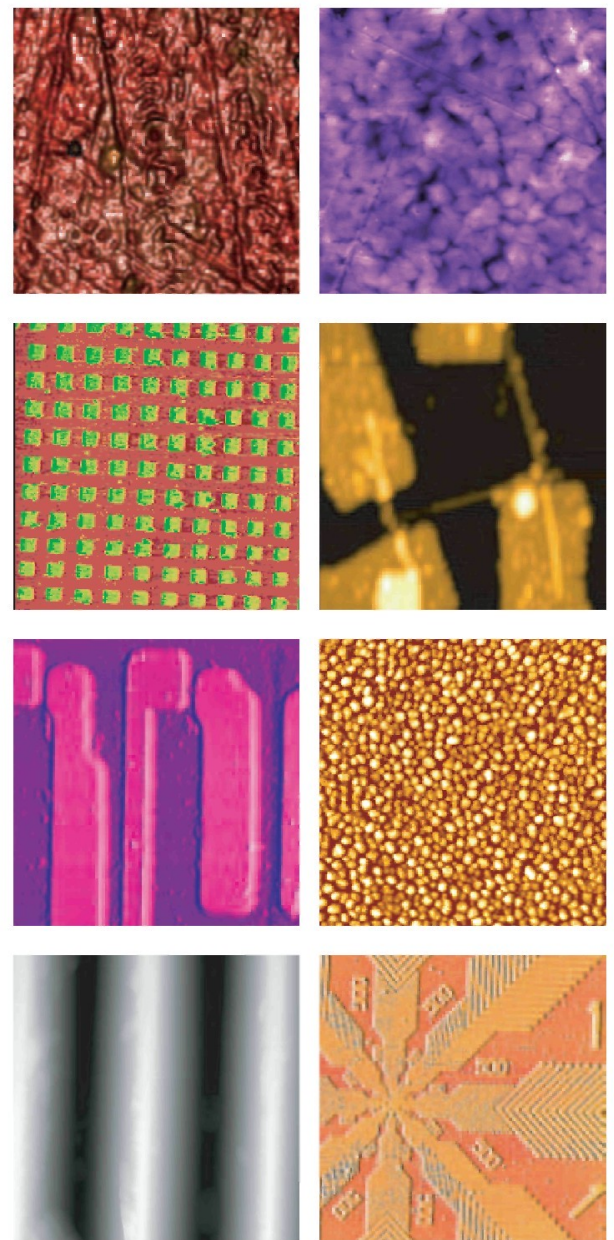

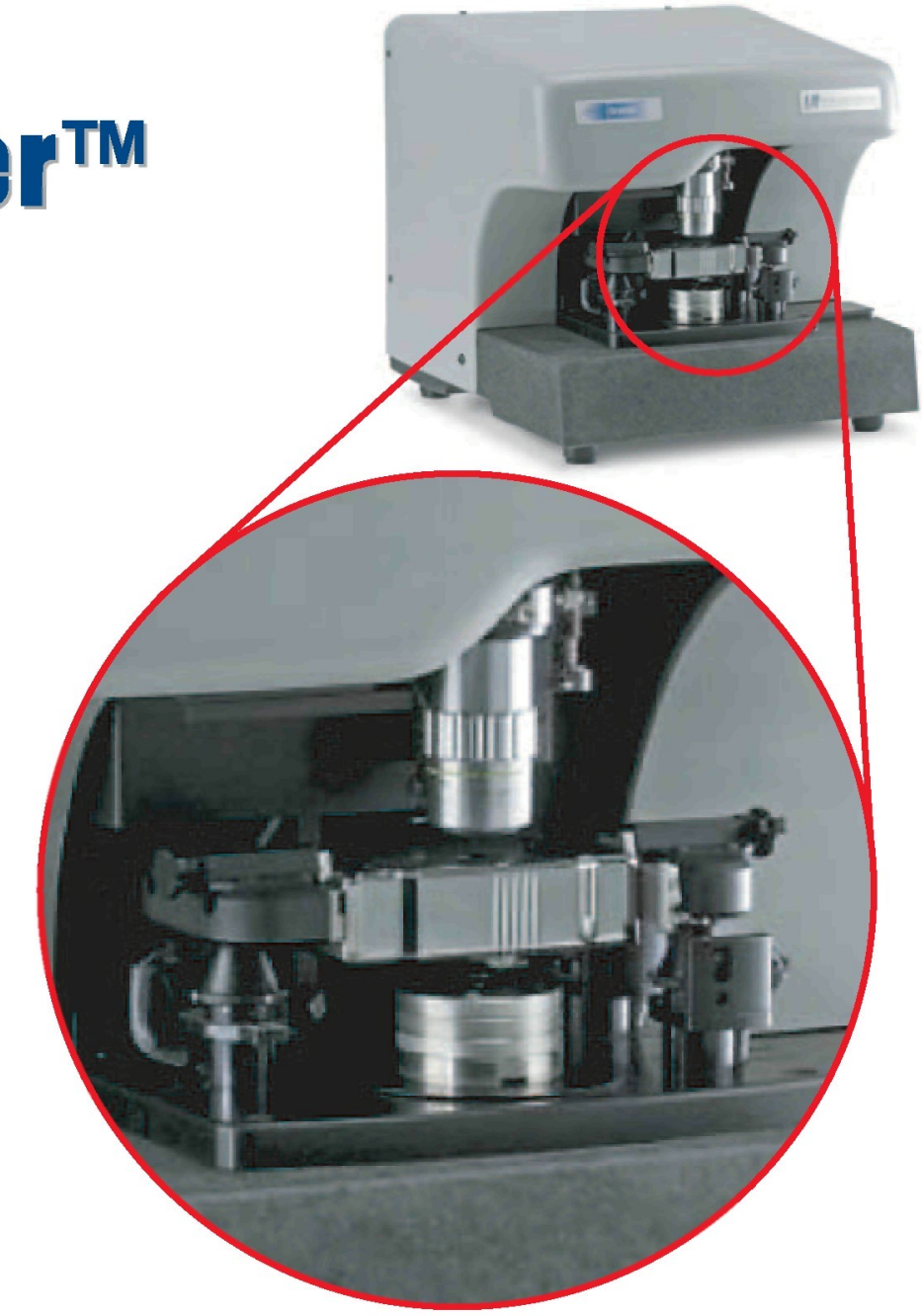

- Nano-R ${ }^{\mathrm{TM}}$ has a new scanner which makes topographic imaging simple

- Crystal Scanner uses Point \& Scan ${ }^{\text {TM }}$ technology load the sample, the probe and just scan

- No complex operating procedures - no laser to adjust

- Motorized x,y stage and automated video microscope features make high resolution nanoscale imaging easy

(800) 246-3704

3350 Scott Blvd. \#29 - Santa Clara, CA 95054

Phone: (408) 982-9492 - Fax: (408) 982-9151

www.pacificnano.com • www.probestore.com 


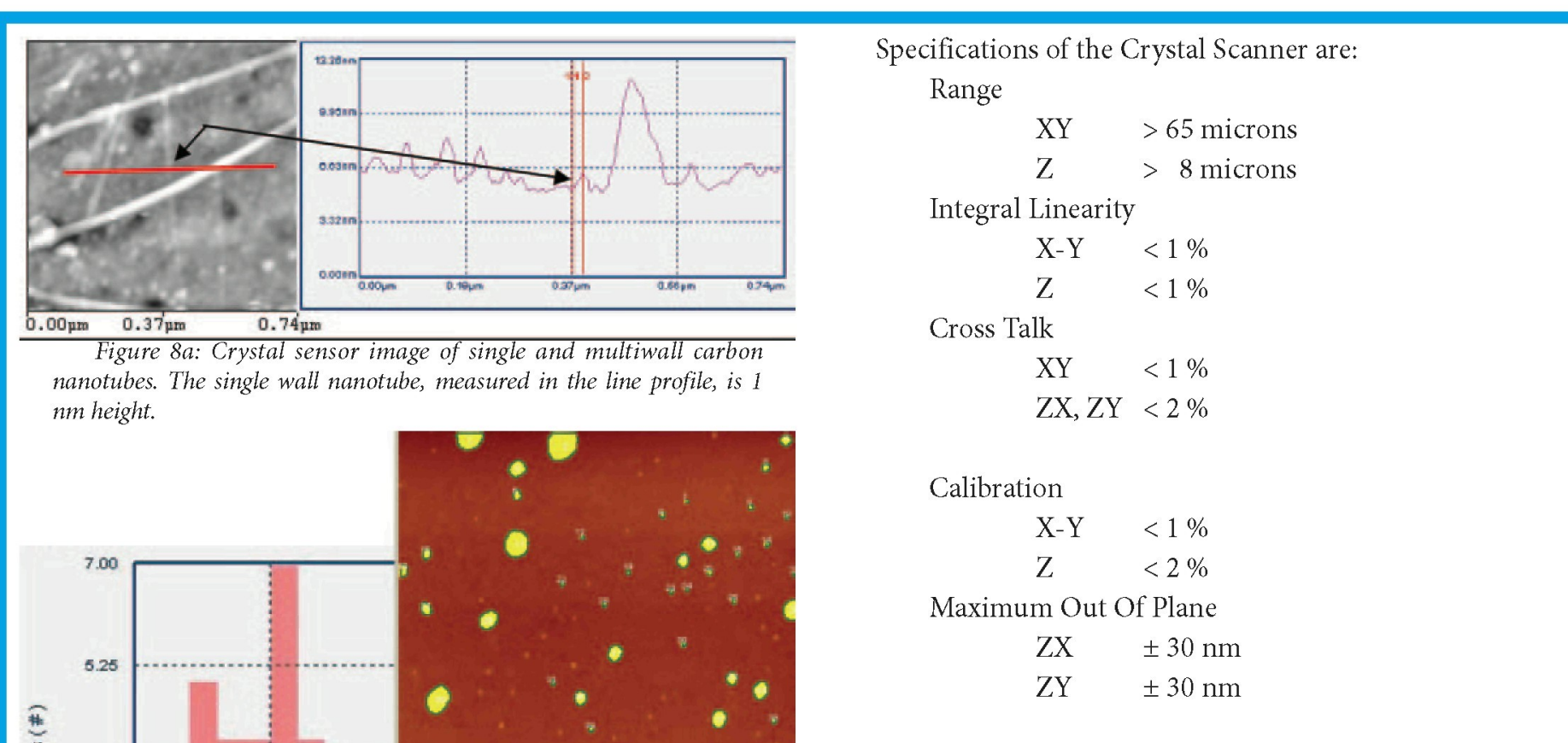

Figure $5 c X$ and $Y$ nonlinearity are determined by comparing the expected position of features on a test pattern with the measured position of features on the test pattern. This figure shows the results form a measurement of the features in the $\mathrm{X}$ axis of a VLSI standard.

\section{Metrology Examples}

Great care must be taken to properly make metrology measurements with scanning probe microscopes. Knowledge of the scanner specifications and probe geometry are essential for making SPM dimensional or metrological measurements. The metrological measurements such as step heights, surface roughness, particle size, and pitches are required for several types of product development, process development, and research areas.

\section{Technical Applications:}

Optical Media - The development of optical storage media, such as digital video disks, requires control of the physical dimensions at the nanometer level. Parameters such as bit sidewall angles, piezoelectric scanners. The optimal scanner for X-Y motion, used in the Pacific Nanotechnology Crystal Scanner, uses a flexure for both axes. This is because a flexure scanner has minimal bow, and X-Y crosstalk. In the $\mathrm{Z}$ axis, a direct piezoelectric drive is used. The direct drive ceramic facilitates viewing of the probe with a video optical microscope. Figure $3 \mathrm{~b}$ illustrates the probe mounting fixture in a Crystal Scanner.

Probes- Probes used in the crystal sensor are chemically fastened to the extended tine of the crystal resonator. This means that any type of probe can be used with the crystal sensor. For example, as illustrated in figure 4 , a tungsten probe or a silicon probe can be used. In the case of a tungsten probe, the angle of the probe is variable.

Specifications- Specifications for the Crystal
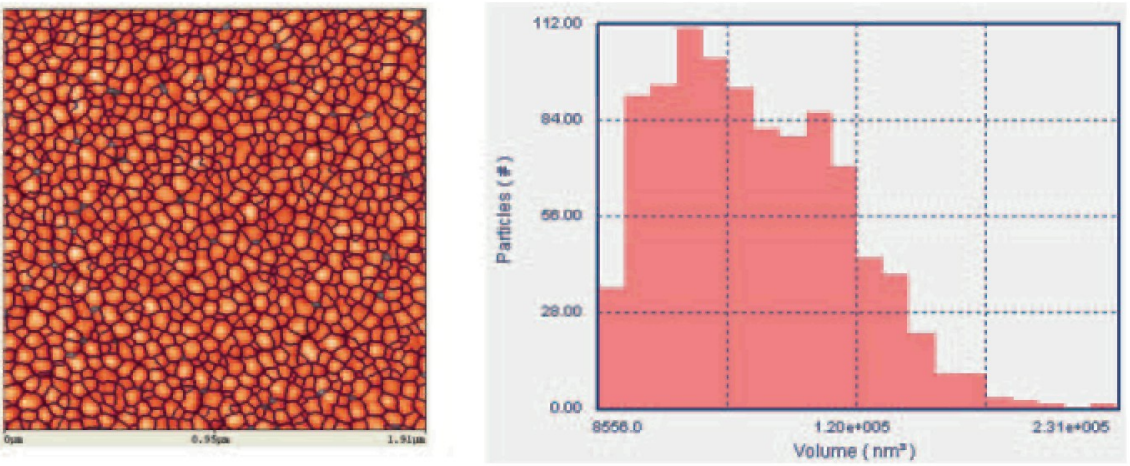

Figure 9: Using specialized software the grains size distribution of this polysilicon sample is measured with the Crystal Scanner. The grain area, perimeter, and volume may be calculated. On the right is a histogram plot for the grain size volume.

Scanner are optimal for making metrological measurements. The specifications are helpful in determining if an application may be possible with the scanner. However, it is important to fully understand the application before the exact application is done. Figures $5 \mathrm{a}-\mathrm{c}$ show some of the data and images used for establishing the specifications of a Crystal Scanner.

bit width, bit length, and bit height must be critically controlled. Figure 6 shows crystal images of DVD bits and the types of analysis that is possible

Flat Panel Displays - It is important to critically measure and control thin film thicknesses in flat panel displays. Because the Crystal Scanner is calibrated in the $\mathrm{X}, \mathrm{Y}$ and $\mathrm{Z}$ axes, it is ideal for 
step height measurements of films used for flat panel displays, see figure 7.

Semiconductor Devices - Characterizing the geometries of structures used in devices is important for improving device density and performance. The Crystal Scanner is ideal for measuring the geometry of semiconductor devices.

Nano Structure Applications:

Nanotubes - Nanotubes play an ever important role in the evolution of nano-scale science and technology. The Crystal Scanner is ideal for both visualizing nanotubes but also measuring their diameter and their length, Figure 8a

Nanoparticles - Characterizing the size and shape of nanoparticles is often done with FE-SEM or even TEM. The Crystal Scanner affords an alternative to these electron beam techniques. With the Crystal Scanner and special software, the diameter, and diameter distribution of nanoparticles is easily measured, figure $8 \mathrm{~b}$

Nano Grains - Grains at the nano-scale play a critical role in the electrical characteristics and mechanical properties of many thin films. Because the images measured with the Crystal Scanner are three dimensional, software can readily count the number and calculate the area and area distributions of grains, see figure 9.

\section{Conclusion}

The Crystal Scanner is optimized for making metrological measurements. The types of measurement that are possible include surface texture, dimensional measurements on micro/nanofabricated devices, nanostructure dimensions, and particle/grain analysis. Such measurements are useful in high technology industries, basic industries and research institutions working in nanotechnology.

\section{The Leaders in Lift-Out Technology}

Now Selling $4^{\text {th }}$ Generation Ex-Situ Nanomanipulator Systems

If you have more than one FIB, just one of our Ex-Situ systems will accomodate them all!

\section{The Ex-Situ Method}

- The "Original" Lift-Out Over 75 Systems Technique

World wide

- Allows your FIB to do what it does best:

- Proven, Fast, Easy Make TEM Samples!

Technology

- Cost Effective

- $95 \%$ Success Rate

Micro Optics of Florida, Inc. (800) 545-3996 info@microopticsfl.com
Quality Preparation

\section{Quality Results}

\section{Advanced EM Specimen Preparation Instuments}
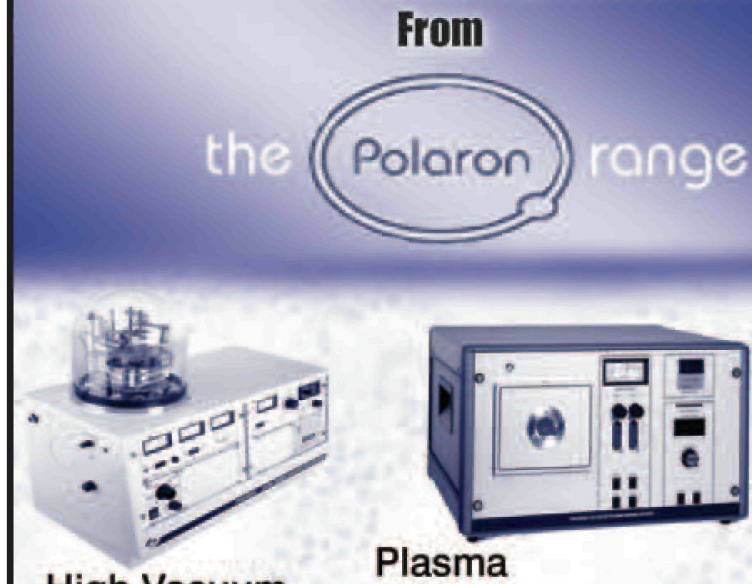

\section{High Vacuum Evaporation}

\section{Etching/ashing \\ ing}

Plasma

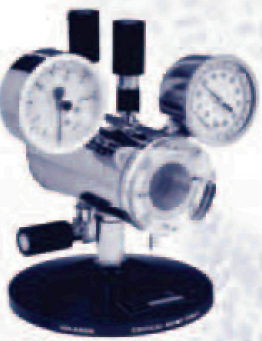

Critical Point Drying
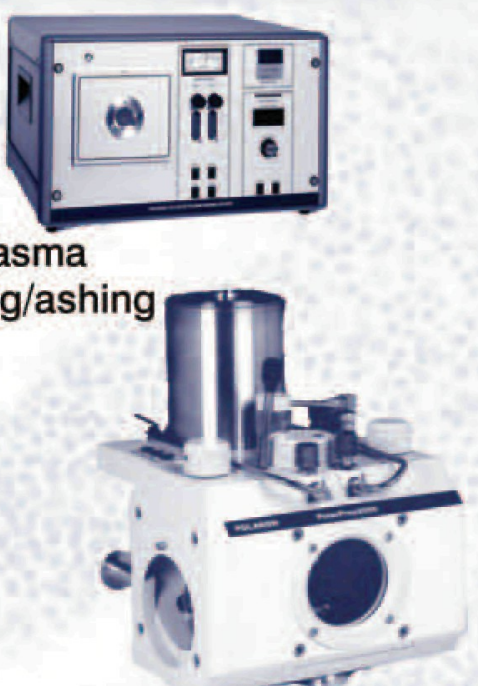

Cryo-SEM

\section{Introducing the} E6500 Evaporator Low Gost - Full Features - Revolutionary Design
ADDING BRILIIANCE TO YOUR VISION

800-992-9037 or 413-786-9322

email: ebs@ebsciences.com 